Informes de la Construcción Vol. 62, 519, 47-53 julio-septiembre 2010 ISSN: 0020-0883 elSSN: 1988-3234 doi: 10.3989/ic.09.063

\title{
La técnica DInSAR: bases y aplicación a la medición de subsidencias del terreno en la construcción
}

\section{DInSAR technique: basis and applications to terrain subsidence monitoring in construction works}

$\underline{\text { E. Sillerico }}^{(*)}$, M. Marchamalo, J. G. Rejas, R. Martínez

\section{RESUMEN}

Las subsidencias se definen como movimientos lentos y paulatinos de la superficie del terreno natural o construido y que pueden afectar a todo tipo de terrenos. Son debidos a cambios tensionales inducidos en éstos, por: descenso del nivel freático (extracción en acuíferos), minería subterránea (minerales, carbón, sal), excavación de túneles, extracción de petróleo o gas, procesos de disolución y lavado de materiales, consolidación de suelos blandos y orgánicos, etc. El control de subsidencias del terreno es esencial en fase constructiva y de monitorización de obras, ya que como se producen de manera progresiva, pueden ser reconocidas antes de que causen daños a la infraestructura. Los procedimientos más habituales para monitorizar subsidencias en obras de ingeniería son la topografía de precisión (nivelación), inclinómetros, fotogrametría, láser escáner y DGPS (Sistema de Posicionamiento Global Diferencial). En la última década se han realizado experiencias con una nueva técnica de medida de subsidencias en la superficie del terreno, a partir de imágenes adquiridas desde satélite por sensores activos en la región de las microondas, denominada DInSAR (Interferometría Diferencial con Radar de Apertura Sintética), y se fundamenta en la detección de pequeñas variaciones de altitud a partir del cálculo de la diferencia de fase de pares de imágenes radar sobre un mismo área de estudio. En este artículo se presenta la evolución de DInSAR, su aplicación a distintos campos con resultados satisfactorios y se expone una revisión sobre su utilización para monitorizar y medir subsidencias en estructuras de tierra en el sector de la Construcción y en los campos de la Geotecnia, Hidrogeología y Vulcanología.

Palabras clave: subsidencias, teledetección, DInSAR, MDT, construcción.
SUMMARY

Subsidences are defined as slow and gradual movements of the terrain or built surface. These may affect all types of terrains, and are caused by tension-induced changes for many reasons, such as lowering water tables (groundwater extraction), underground mining (minerals, coal, salt), excavation of tunnels, extraction of oil or gas, slow processes of dissolution and lixiviation of materials, consolidation of soft soils, organic soils,... The measurement and monitoring of land subsidences are the major components of infrastructures' auscultation in the construction and monitoring phases. In many cases, subsidences occur gradually and may be recognized before they cause damage in the works. The most common procedure for subsidence's control in engineering until now, is based on the use of instrumentation and methodologies for surveying such as precision levelling, inclinometers, photogrammetry, laser scanning and DGPS (Differential Global Positioning System). In the last decade a new technique is emerging, DInSAR (Differential Interferometry Synthetic Aperture Radar), which is based on the detection of small variations in altitude from the phase difference calculation between pairs of radar images (satellite's data acquired from active sensors in the microwave region) covering the same area of study. This article presents the evolution of this technique for measuring surface land subsidence and its application to various fields with satisfactory precision results. It presents a review on the use of DInSAR for monitoring and measuring subsidence of land and earth structures in Construction and in the fields of Geotechnics, Hydrology and Volcanology.

\section{$479-1$}

Keywords: subsidence, remote sensing, DInSAR, DTM, construction.

\footnotetext{
(*) Laboratorio de Topografía y Geomática. Dpto. de Ingeniería y Morfología del Terreno, E.T.S.I. Caminos, Canales y Puertos (U.P.M.), Madrid (España) Persona de contacto/Corresponding author: eleanorsillerico17@hotmail.com (E. Sillerico)
} 
1. Geometría de una adquisición SAR interferométrica. Los parámetros de la figura son los siguientes: B es la línea de base, B I es la base perpendicular, $\mathrm{M}$ y $\mathrm{S}$ son los satélites maestro y esclavo, $R$ es la distancia del satélite Ma P, y z es el movimiento o deformación vertical.

\section{LA INTERFEROMETRÍA SAR Y LA INTER- FEROMETRÍA DIFERENCIAL SAR (DInSAR)}

La Interferometría SAR (INSAR) y su variante, DInSAR, son técnicas basadas en el proceso y análisis de imágenes de Radar de Apertura Sintética (SAR). Los SAR son sensores de los denominados activos, que registran imágenes de alta resolución espacial. Una antena de apertura sintética o virtual, consiste en un vector de sucesivas y coherentes señales de radar que son transmitidas y recibidas por una pequeña antena que se mueve a lo largo de un determinado recorrido de vuelo u órbita. El procesamiento de la señal usa las magnitudes y fases de la señal recibida sobre sucesivos pulsos para crear una imagen (15).

A partir de interferometría, los datos obtenidos son las distancias entre el satélite y la superficie del terreno, calculados mediante la medición de tiempos y desfases. Las principales plataformas que utilizan radares de apertura sintética se muestran en la Tabla 1. En síntesis los SAR (Synthetic Aperture Radar) son sistemas de radares que generan imágenes de alta resolución (1).

La interferometría SAR es una técnica de procesado de imagen que permite, a partir de 2 pases interferométricos del sensor SAR, generar el Modelo Digital de Elevaciones (MDE) y la coherencia relativa, entre otras aplicaciones.

Así pues InSAR consiste en medir las diferencias de fase entre el satélite y la tierra en dos pasos del satélite por la misma zona (6).

Estas diferencias de fase están en función de la distancia y pueden ser debidas básicamente a dos motivos: en primer lugar, a la diferencia de posición entre las dos trayectorias del satélite, hecho que permite medir la topografía del terreno, como se explicará a continuación; en segundo lugar, a un desplazamiento de la zona observada entre las dos adquisiciones, que puede ser causado por varios fenómenos, como por ejemplo, un terremoto o una subsidencia del terreno debida a la construcción de un túnel. De esta forma, "la interferometría SAR (InSAR) es una técnica que permite la detección de movimientos y deformaciones de la superficie terrestre" (Martínez, 2005) (12).

La señal reflejada se ve afectada por varios parámetros como son la atmósfera, el ruido y los movimientos en la superficie. En el caso de movimientos o deformaciones en la super-

Tabla 1

Características de los principales sensores SAR

\begin{tabular}{|l|c|c|c|c|c|c|c|c|}
\hline \multicolumn{1}{|c|}{ Satélite } & $\begin{array}{c}\text { Longitud de } \\
\text { onda [cm] }\end{array}$ & Polarización & $\begin{array}{c}\text { Ángulo de } \\
\text { incidencia [ }{ }^{\circ} \text { ] }\end{array}$ & $\begin{array}{c}\text { Resolución } \\
\text { espacial [m] }\end{array}$ & $\begin{array}{c}\text { Ciclo de } \\
\text { repetición [dias] }\end{array}$ & $\begin{array}{c}\text { Periodo } \\
\text { orbital }\end{array}$ & $\begin{array}{c}\text { Altitud de } \\
\text { órbita [km] }\end{array}$ & País \\
\hline ERS-1 & $5-6$ [c-band] & $\mathrm{VV}$ & 23,5 & 25 & 3,5 y 176 & 101 min & $782-785$ & Europa \\
\hline ERS-2 & $5-6$ [c-band] & $\mathrm{VV}$ & 23,5 & 25 & 35 & & 780 & Europa \\
\hline JERS-1 & 23,5 [l-band] & $\mathrm{HH}$ & 35 & 18 & 44 & & 568 & Japón \\
\hline RADARSAT & $5-6$ [c-band] & $\mathrm{HH}$ & 37 & 10 & 24 & & $793-821$ & Canada \\
\hline ENVISAT & $5-6$ [c-band] & $\mathrm{VV}$ & 21 [IS2] y 38 [IS5] & 25 & 35 & 100,7 min & 800 & Europa \\
\hline RADARSAT-2 & [c-band] & HH-HV-VV-VH & $20-49$ & $3-100$ & $2-3$ & & 798 & Canada \\
\hline TERRASAR-X & $3,10[x-b a n d]$ & HH-HV-VV-VH & $20-45$ & $1-18$ & 11 & 94,92 min & $512-530$ & Alemania \\
\hline
\end{tabular}

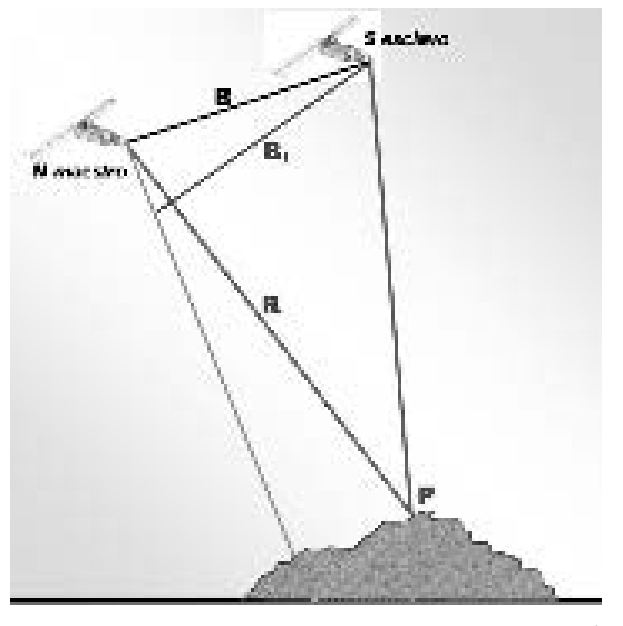

ficie (Figura 1), la metodología de cálculo es la siguiente: se tiene un punto $P$ que se ha desplazado a un punto $\mathrm{P}^{\prime}$ durante el tiempo entre una adquisición y la otra. De esta forma la fase interferométrica se ve afectada por varios parámetros, según se presenta en la siguiente ecuación:

$$
\begin{aligned}
& \phi_{\text {Int }}=\phi_{S}-\phi_{M} \\
& \phi_{\text {Int }}=\frac{S P-M P}{\frac{\lambda}{4 \cdot \pi}}+\frac{S P^{\prime}-M P}{\frac{\lambda}{4 \cdot \pi}}+\phi_{\text {Atm }}+\phi_{\text {Ruido }} \\
& \phi_{\text {Int }}=\phi_{\text {Topo }}+\phi_{\text {Mov }}+\phi_{\text {Atm }}+\phi_{\text {Ruido }}
\end{aligned}
$$


Donde:

$\phi_{\mathrm{s}}$ y $\phi_{M}$ son las fases interferométricas de la posición del satelite esclavo y maestro.

$\phi_{\text {Atm }}$ es la contribución de la atmósfera,

$\phi_{\text {Ruido }}$ componente debida al ruido,

$\phi_{\text {Topo }}$ componente debida a la topografía del terreno,

$\phi_{\text {Mov }}$ componente debida al movimiento o deformación,

SP es la distancia del satélite esclavo al punto, siendo $\mathrm{P}^{\prime}$ el punto desplazado,

MP es la distancia del satélite maestro al punto y

$\lambda$ es la longitud de onda radar

La interferometría SAR diferencial (de siglas $\boldsymbol{D}$-InSAR), es una variante de la anterior. Se trata de una técnica de proceso de imagen que permite la generación de mapas de desplazamiento del terreno y el cálculo de la coherencia relativa, a partir de $n$ pasos del sensor SAR por una misma zona de estudio (11).

El análisis D-InSAR puede ser multitemporal, es decir, que se adquieren los pares de imágenes a lo largo del tiempo, para seguir movimientos de varios meses o años. Existen distintas técnicas para el procesado D-InSAR; por ejemplo, la obtención de los desplazamientos se realiza a partir de las zonas alejadas del fenómeno (volcán, falla, deslizamiento), zonas que se suponen libres de cambios y con geometría constante, lo que permite filtrar ciertas diferencias "no reales", producidas por cambios atmosféricos. A partir de las zonas alejadas se va "integrando" hacia el centro de la zona de estudio, obteniendo los desplazamientos totales.

Con la técnica de interferometría diferencial DInSAR se puede eliminar el inconveniente planteado en InSAR debido a que la deformación se encuentra mezclada con la señal topográfica, ya que se despeja ésta del interferograma, obteniéndose el interferograma diferencial SAR.

La técnica DInSAR se utiliza para detectar y medir movimientos muy pequeños de la superficie. Para poder detectar deformaciones tan pequeñas hay que deshacerse de la componente de topografía. Después de descomponer la fase obtenida se calcula la magnitud del movimiento. Dada la pequeña longitud de onda en la que se opera, se detectan movimientos subcentimétricos.

\section{REVISIÓN DE LA TÉCNICA DInSAR APLI- CADA AL CÁLCULO DE SUBSIDENCIAS}

De acuerdo a la bibliografía consultada, en este apartado se presentan gráficos resumen de las aplicaciones de la técnica DInSAR, en diferentes campos y en distintos países.

La Figura 2 muestra los años de publicación de los artículos consultados sobre la aplicación práctica de la técnica DInSAR. Tal y como se puede observar, en la última década ha tenido un gran crecimiento y, sobre todo, a partir del año 2003

Del análisis de este gráfico se deduce que la aplicación de esta técnica presenta una clara tendencia ascendente y que la medición de subsidencias se irá optimizando debido al perfeccionamiento tecnológico de los sensores.

En el gráfico de la Figura 3 se muestran los porcentajes que representan a los distintos campos de aplicación de la técnica DInSAR.

Se observa que en la última década, la investigación de la aplicación de la técnica DInSAR se ha concentrado principalmente en dos campos: los asentamientos debidos a la extracción de agua (2) (8) (16) (18) (19) del subsuelo y a la actividad volcánica (3) (10) (13) (17).

A continuación el campo de mayor aplicación es el de las deformaciones y subsidencias debidas a los sismos (2) (7), a la minería en
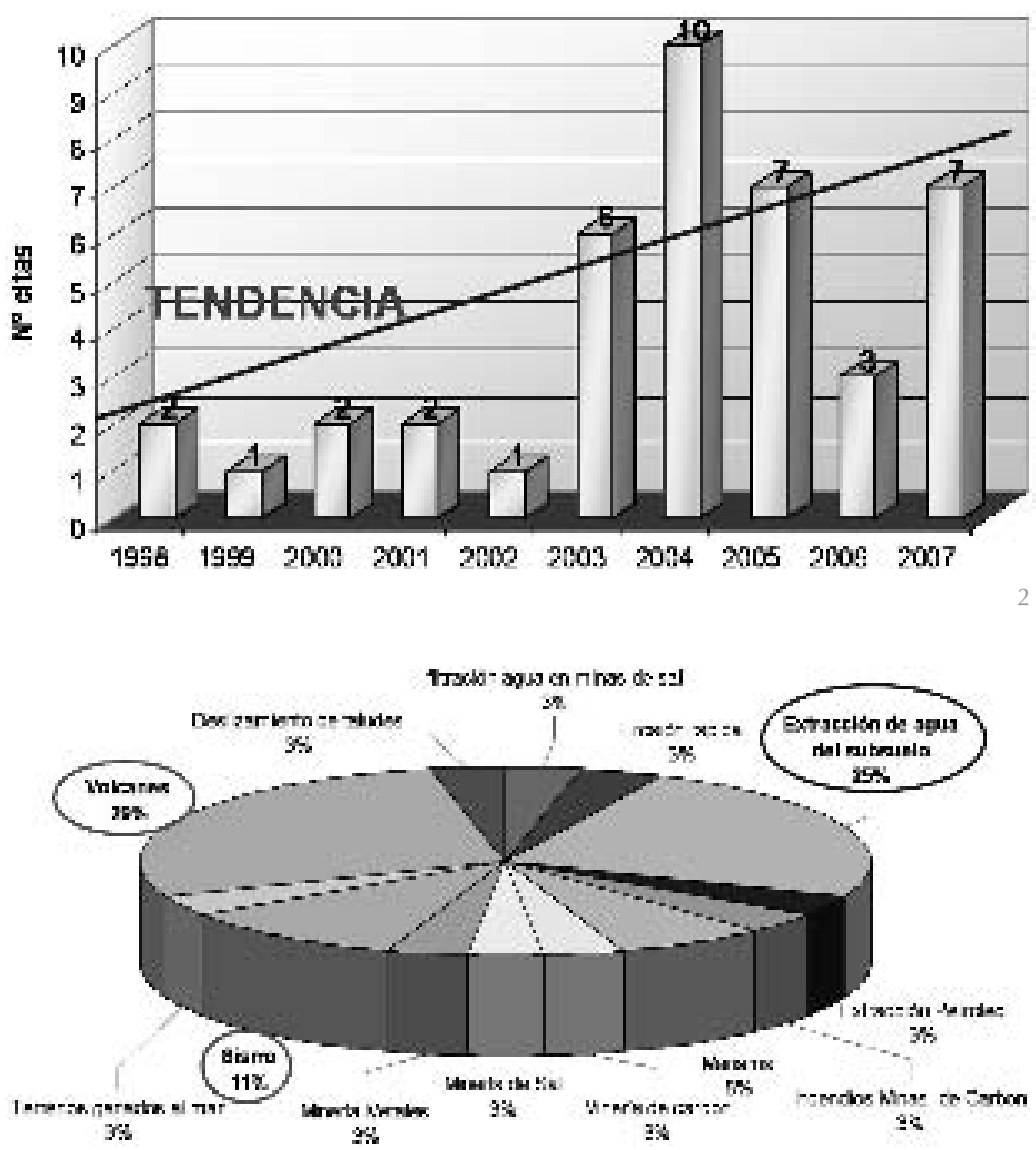
4. Porcentaje del tipo de satélites utilizados en las distintas aplicaciones. general (2) (4) (9) (14) y a los deslizamientos de ladera (5) (20). Los demás porcentajes se presentan de forma homogénea.

\section{PLATAFORMAS UTILIZADAS EN LA ACTUALIDAD}

Uno de los satélites de última generación es el satélite Envisat (Environmental Satellite) que es de observación terrestre, construido por la Agencia Espacial Europea (ESA). Fue lanzado el 1 de marzo de 2002 en un cohete Ariane 5 en una órbita polar síncrona con el Sol a una altura de $790 \mathrm{~km}(+/-10 \mathrm{~km})$. Orbita la Tierra en un periodo de cerca de 101 minutos con un periodo de repetición de ciclos cada 35 días. El Envisat es el mayor observatorio de la atmósfera y superficie terrestre lanzado en los últimos años. Este satélite esta equipado con el sensor ASAR.

Anteriormente la ESA lanzó el European Remote Sensing Satellite (ERS-1), él primer satélite de observación de la Tierra. Fue lanzado el 17 de julio de 1991, por un Ariane 4 desde Kourou (Guyana Francesa), en una órbita polar síncrona con el sol a una altura de entre 782 y 785 km.

Su sucesor, ERS-2, fue lanzado el 21 de abril de 1995 también por un Ariane 4 desde Kourou. Es prácticamente igual al ERS-1, pero añadiendo el GOME (Global Ozone Monitoring Experiment) y un espectrómetro de absorción. Cuando ERS-2 fue lanzado, compartía el mismo plano orbital con el ERS-1, lo cual posibilitó una misión de "tándem" con el ERS-2, pasando un día después por la misma zona que el ERS-1. Esto facilitó el estudio de la aplicación de la técnica DInSAR a la medición de pequeñas deformaciones en la superficie terrestre.

JERS-1 es un satélite de observación para cubrir la superficie terrestre mundial, orientado a la observación alrededor del planeta y la explotación de los recursos. Fue lanzado en una órbita solar síncrona a una altitud de $568 \mathrm{~km}$ con un período recurrente de 44 días por el vehiculo $\mathrm{H}$-I de lanzamiento el $11 \mathrm{de}$

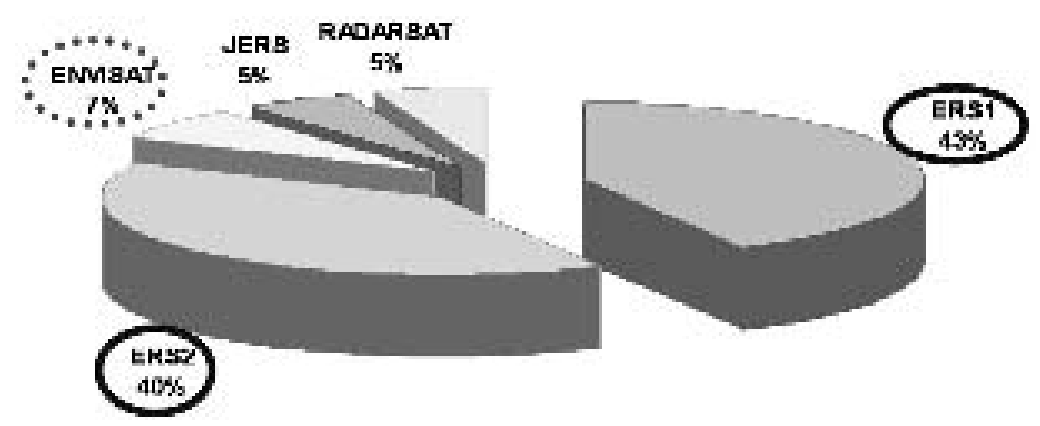

febrero de 1992 por el Organismo Nacional de Actividades Espaciales del Japón (NASDA) Centro Espacial de Tanegashima, y continúa recogiendo datos de alto rendimiento, equipado con Radar de Apertura Sintética (SAR) y Sensor Óptico (OPS).

El satélite RADARSAT- 1 fue lanzado el 4 de noviembre de 1995, es el resultado de un consorcio entre el Gobierno Canadiense, la industria privada y la NASA. Su órbita heliosincrónica tiene un ciclo repetitivo de 24 días. Proporciona diariamente imágenes regulares sobre el ártico, y cada cinco días sobre latitudes ecuatoriales. El SAR, con el que esta equipado, utiliza únicamente la banda $\mathrm{C}$ de una sola frecuencia $(5.3 \mathrm{GHz})$ y puede dirigir el haz del radar hasta un alcance de $500 \mathrm{~km}$.

El 14 de diciembre de 2007, fue lanzado su sucesor, el RADARSAT-2, desde Baikonur, Kazajstán por el trasbordador Soyuz y se prevé que alcance una vida útil mínima de 7 años.

La Figura 4 recoge el porcentaje de utilización en la aplicación DinSAR de los distintos sensores SAR satelitales disponibles. Se observa que los datos que más información han aportado provienen de los satélites ERS-1 y ERS-2 debido, entre otras causas, a la antigüedad de éstos.

\section{VIABILIDAD Y FIABILIDAD}

En este apartado se analiza la viabilidad y la fiabilidad de la técnica DInSAR en la medida de subsidencias en la superficie terrestre a la luz de los datos obtenidos en la fase de análisis. En el articulo "A land subsidence study via DInSAR technique over large urbanized areas", Cascini L., Ferlisi S., Peduto D., Di Nocera S., Fornaro G., Serafino F (5), registraron como mínima deformación en valor absoluto $|\Delta z|=1,5 \mathrm{~mm}$, no obstante los autores consideran que si ésta era menor de $2 \mathrm{~mm}|\Delta z| \leq 2,0$ $\mathrm{mm}$, se consideran los píxeles estables y si por el contrario fuera mayor de $|\Delta z|>2,0 \mathrm{~mm}$, se debe plantear una investigación sobre las causas de la subsidencia.

Una de las ventajas más notables de la técnica DInSAR y que la hace técnicamente viable es que, a diferencia de las técnicas tradicionales, puede cubrir grandes áreas (hasta $10.000 \mathrm{~km}^{2}$ ), facilitando la recolección y procesamiento de gran cantidad de datos con un bajo coste.

La posibilidad de detectar subsidencias de orden subcentimétrico hace que la técnica sea viable en la monitorización de casi cualquier estructura de tierra que esté sujeta a desplazamientos. No obstante, la técnica 
se basa en la extracción de la información de un par de imágenes adquiridas en distintos lapsos de tiempo (espacio de tiempo suficiente para notar una deformación del suelo), por lo que puede ocurrir que la frecuencia de muestreo de una auscultación corriente sea superior a la frecuencia de adquisición de imágenes; por tanto no se ha estudiado la viabilidad de aplicar la técnica a obras en las que se necesiten contar con datos diarios.

En estos casos la técnica DInSAR podría ser aplicable al control complementario al que se realiza con topografía convencional o DGPS según las conclusiones expuestas en la página oficial de la ESA (European Space Agency) acerca de la extensión de la línea Jubilee del Metro de Londres, donde se registraron subsidencias del orden de 7 mm/año (Figura 5). Estas mediciones se realizaron entre 1995 y 2000, durante los 5 años que duraron los trabajos de construcción del túnel.

De acuerdo a lo descrito anteriormente, se llega a los siguientes enunciados sobre la aplicación de la técnica DInSAR en la construcción:

- La técnica DInSAR es viable en varios campos de aplicación ya que se ha demostrado su potencial de monitorización de velocidades de deformación subcentimétrica.

- La técnica DInSAR permite cubrir grandes áreas (hasta $10.000 \mathrm{~km}^{2}$ ), con un coste bajo por hectárea.

- En la etapa constructiva, es una técnica complementaria de auscultación siempre que el periodo de construcción sea suficientemente extenso, como para poder contar con la cantidad de información necesaria para emitir un informe consistente.

- En la etapa de monitorización de la obra, DInSAR tiene gran potencial de uso debido a la obligatoriedad de monitorizar la obra entre 5 y 15 años después de finalizada, a su bajo coste por hectárea y al registro de movimientos en áreas extensas aledañas a la obra.

- En la bibliografía consultada se ha podido observar que los resultados obtenidos mediante DInSAR han sido contrastados con otros métodos tradicionales de monitorización de subsidencias como el GPS y la nivelación geométrica de precisión; así, las mediciones registradas mostraban resultados con escasa variación entre técnicas.

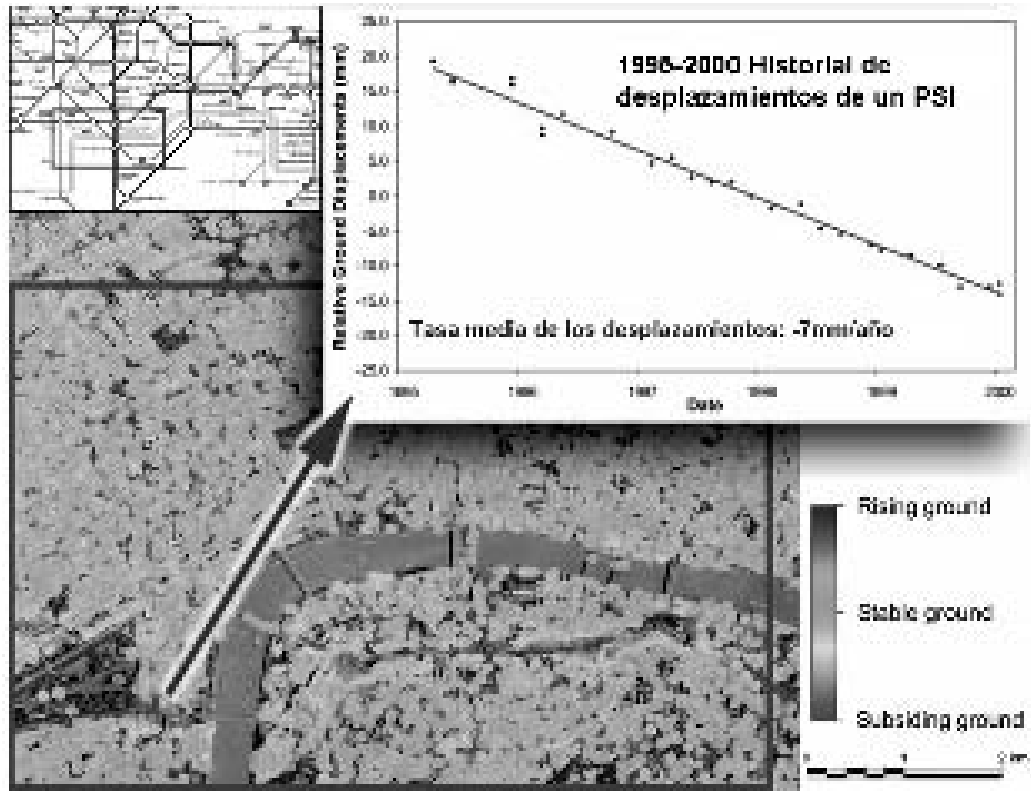

Como desventajas se puede enumerar las siguientes:

- Puede ocurrir que la frecuencia de muestreo de una auscultación corriente sea superior a la frecuencia de adquisición de imágenes para estudiar; por tanto no se ha estudiado la viabilidad de aplicar la técnica a obras en las que se necesiten contar con datos diarios.

- Ocurrencia de algunos problemas técnicos a la hora de obtener las imágenes satelitales SAR. Por ejemplo los problemas técnicos orbitales que afectaron al ERS-2 en febrero de 2001.

\section{EVOLUCIÓN DE LOS MÉTODOS PARA MEDIR DEFORMACIONES DEL TERRENO}

La técnica InSAR ha evolucionado con el tiempo y además de la técnica DInSAR se pueden describir las siguientes evoluciones y técnicas derivadas:

\subsection{PSI Persistent Scatter InSAR (InSAR de Dispersión Persistente)}

PSI hace mediciones de movimientos del terreno en puntos de dispersión persistentes. Los puntos de dispersión persistente se seleccionan previamente y se caracterizan por ser tales como los tejados de edificios, estructuras metálicas y elementos naturales destacados y permanentes. En las zonas urbanas, puede haber 600 puntos de dispersión persistente por kilómetro cuadrado. Excepcionalmente, esta técnica ofrece la posibilidad de obtener un registro de movimientos históricos de más 
de una década para cada punto de dispersión persistente individual.

PSI utiliza datos adquiridos de satélites radar como el ERS-1, ERS-2, Envisat y Radarsat. Todo el Reino Unido, la mayor parte de la Europa continental y gran parte de los EE.UU. y Japón tienen suficientes datos para permitir el proceso de la información PSI, con las imágenes adquiridas con regularidad. Debido al gran volumen de datos, la superficie máxima procesable en un bloque es actualmente de 50 x 50 kilómetros.

PSI filtra los errores inducidos por la atmósfera y la topografía (en relación a determinadas condiciones climáticas y la precisión de los modelos terrestres) mediante la utilización de al menos 30 escenas para calcular una corrección atmosférica de los interferogramas.

\subsection{CRInSAR Corner Reflectors InSAR (InSAR con reflectores de esquina)}

Para las mediciones en puntos específicos y predeterminados, se puede hacer uso de de los reflectores de esquina de bajo coste, actuando como puntos de dispersión permanentes artificiales construidos a propósito. El trabajo del grupo NPA ${ }^{1}$ ha contribuido al desarrollo de los "Reflectores de esquina INSAR" como una herramienta ideal para supervisar el desplazamiento de obras de ingeniería tales como presas, puentes, oleoductos, edificios, o incluso zonas de inestabilidad de laderas.

\subsection{CATInSAR Compact Active Transponders (Transmisores activos de tipo compacto)}

Actualmente se han desarrollado los transmisores compactos activos (CATs), que son dispositivos automáticos que transmiten una señal de radar hacia la antena radar del satélite que está en órbita. Esta transmisión es registrada por el satélite de la misma manera que un haz se refleja en un reflector de es- quina, y permite la misma medición precisa del desplazamiento del terreno. Los CATs son más sencillos de instalar que los reflectores de esquina, son menos vulnerables estructuralmente, y suponen un considerable ahorro de costes. Actualmente, están siendo evaluados por el grupo NPA como sucesores naturales de los reflectores de esquina para la medición de puntos específicos.

\section{CONCLUSIONES}

Las distintas publicaciones científicas consultadas y los constantes trabajos que se presentan en eventos internacionales demuestran que la técnica DInSAR es viable y fiable para medición de subsidencias continuas por su precisión subcentimétrica debido a sus fundamentos físicos y matemáticos.

Es económicamente competitiva a partir de un umbral de superficie; y es recomendable su aplicación especialmente en la fase de monitorización, siendo complementaria en la fase de construcción.

Se puede emplear la técnica para la evaluación de la fiabilidad de estructuras de tierra como presas de materiales sueltos, movimiento de taludes o incluso varias estructuras al mismo tiempo, siempre y cuando los movimientos sean lo suficientemente lentos.

Actualmente se cuenta con un historial de más de 17 años de imágenes del globo terrestre adquiridas por sensores SAR, las cuales están disponibles para ser procesadas y por tanto para calcular subsidencias del terreno.

Este documento constituye un avance de la línea de investigación realizada en el Laboratorio de Topografía y Geomática de la E.T.S.I. de Caminos, Canales y Puertos de Madrid sobre teledetección con sensores activos aplicada a la ingeniería civil y la construcción.
${ }^{1}$ El grupo NPA es una empresa consultora experimentada en observaciones satelitales de la tierra. [www.npagroup.com].

\section{BIBLIOGRAFÍA}

(1) Agudo, M., Biescas, E., Monserrat, O., Martínez, J., Crosetto, M., Herrera, G.: “¿Cómo medir las deformaciones del terreno con teledetección radar?", 6ª Semana de Geomática, Barcelona-España, 2003.

(2) Biescas, E., Agudo, M., Monserrat, O., Ibañez, C., Crosetto, M.: "Aplicaciones de la interferometría SAR para la medida de deformaciones del terreno". Instituto de Geomática, Castelldefels, España, 2003.

(3) Bonaccorso, A., Sansosti, E., Berardino, P.: "Comparison of Integrated Geodetic Data Models and Satellite Radar Interferograms to Infer Magma Storage during the 1991-1993 Mt. Etna Eruption". Pure Appl. Geophys", 161, pp. 1345-1357, 2004.

(4) Carnec C., C. Delacourt.: "Three years of mining subsidence monitored by SAR interferometry, near Gardanne, France", Journal of Applied Geophysics 43 (2000), pp. 43-54, 1999.

(5) Cascini, L., Ferlisi, S., Peduto, D., Di Nocera, S., Fornaro, G., Serafino, F.: "A land subsidence study via DInSAR technique over large urbanised areas", Urban Remote Sensing Joint Event, Paris, 2007.

(6) Crosetto, M., Crippa, B., Biescasa, E.: "Early detection and in-depth analysis of deformation phenomena by radar interferometry", Engineering Geology 79 (2005) 81-91, 2004

(7) Ding, X.L., Liu, G.X., Li, Z.W., Li, Z.L. and Chen, Y.Q.: "Ground Subsidence Monitoring in Hong Kong with Satellite SAR Interferometry", Photogrammetric Engineering \& Remote Sensing. Vol. 70, No. 10, (2004), pp. 1151-1156, 2004 
(8) Gili Ripoll, J. A.: "Instrumentación para control de movimientos de ladera: sistema GPS y otros, Operatividad de la instrumentación en aguas subterráneas, suelos contaminados y riesgos geológicos", IGME, MADRID. España, 2003.

(9) Herrera, G., Tomás, R., López-Sánchez, J.M., Delgado, J., Mallorqui, J.J., Duque, S., Mulas, J.: "Advanced DInSAR analysis on mining areas: La Union case study (Murcia, SE Spain)", Engineering Geology 90 (2007), pp. 148-159, 2007.

(10) Lagios, E., Sakkas, V., Parcharidis, Is., Dietrich, V.: "Ground deformation of Nisyros Volcano (Greece) for the period 1995-2002: Results from DInSAR and DGPS observations", Bull. Volcanol. (2005) 68: pp. 201-214, 2005.

(11) Zhilin, Li., Zou, W., Ding, X., Chen, Y. and Liu, G.: "A Quantitative Measure for the Quality of INSAR Interferograms Based on Phase Differences", Photogrammetric Engineering \& Remote Sensing. Vol. 70, No. 10, (2004), pp. 1131-1137, 2004.

(12) Martínez Villar, J.: "Elaboración y Análisis de Imágenes Radar desde satélite: Monitorización de deformaciones del Terreno", Instituto de Geomática, Castelldefels, España, 2005.

(13) Massonnet, D., Feigl, K.L.: "Radar Interferometry and its application to changes in the Earth's surface", Reviews of Geophysics, 36, 4 / (November 1998), pp. 441-500, 1998.

(14) Raucoules, D., Colesanti, C., Carnec, C.: "Use of SAR interferometry for detecting and assessing ground subsidence", C. R. Geoscience 339 (2007), pp. 289-302, 2007.

(15) Rosen, P.A., Hensley, S., Joughin, I.R., Li, F.K., Madsen, S.N., Rodríguez, E. and Goldstein, R.M.: "Synthetic Aperture Radar Interferometry", Proceedings of the IEEE, Vol. 88, No. 3, March 2000.

(16) Ud din, S., Al-Dousari, A., Al-Ghadban, A., Aritoshi, M. 2005, "Use of interferometric techniques for detecting subsidence in the oil fields of Kuwait using Synthetic Aperture Radar Data", Journal of Petroleum Science and Engineering , Vol. 50 (2006), pp. 1-10, 2006.

(17) Scharrer, K. \& Spieler, O. \& Mayer, Ch. \& Münzer U.: "Imprints of sub-glacial volcanic activity on a glacier surface-SAR study of Katla volcano, Iceland", Bull. Volcanol. DOI 10.1007/s00445-0070164-z, 2007.

(18) Stramondo, S., Saroli, M., Tolomei, C., Moro, M., Doumaz, F., Pesci, A., Loddo, F., Baldi, Boschi, E.: "Surface movements in Bologna (Po Plain - Italy) detected by multitemporal DInSAR", Remote Sensing of Environment, Vol. 110 (2007), pp. 304-316, 2007.

(19) Strozzi, T., Wegmüller, U., Werner, C.L., Wiesmann, A., Spreckels, V.: "JERS SAR Interferometry for Land Subsidence Monitoring", IEEE Transactions on Geoscience and Remote Sensing, Vol. 41, No. 7, (July 2003), pp. 1702-1708, 2003.

(20) Xia, Y., Kaufmann, H. and Guo, X.F.: "Landslide Monitoring in the Three Gorges Area using D-INSAR and Corner Reflectors", Photogrammetric Engineering \& Remote Sensing, Vol. 70, No. 10, (October 2004), pp. 1167-1172, 2004.

(21) Case study: London Metro Extensión, ESA. [www.altamira-information.com]. 